\title{
Inferencia del capital de trabajo en el desarrollo empresarial
}

\author{
Inference of working capital in business development
}

Inferência do capital de giro no desenvolvimento de negócios

\section{ARTÍCULO GENERAL}

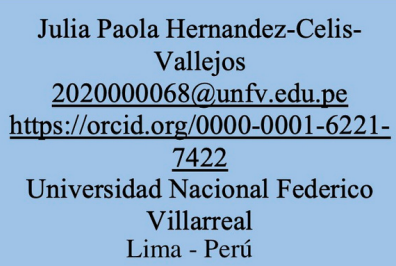

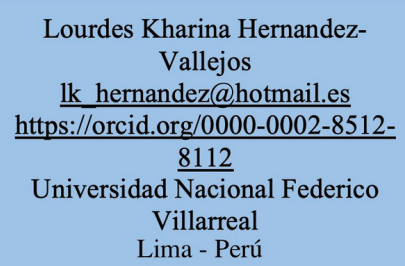

$\begin{gathered}\text { Andrea Del Rocío Hernandez- } \\ \text { Vallejos } \\ \text { andreadelrociohv19@gmail.com }\end{gathered}$
$\frac{\frac{\text { https://orcid.org/0000-0002-0243- }}{2216}}{\frac{2216}{\text { Unional Federico }}}$
$\begin{gathered}\text { Villarreal } \\ \text { Lima - Perú }\end{gathered}$

Domingo Hernandez-Celis domingo.hernandez@uwiener.ed https://orcid.org/0000-00029759-4436

Universidad Nacional Federico Villarreal

Universidad Norbert Wiener Lima - Perú

Recibido 07 de Junio 2021 | Arbitrado y aceptado 25 de Agosto 2021 | Publicado en 04 Diciembre 2021

\begin{abstract}
RESUMEN
El problema se ha identificado en el deficiente desarrollo de las empresas indicadas. Esta problemática se expresa en la siguiente pregunta: ¿Cómo se podrá inferir el capital de trabajo en el desarrollo de las empresas del Centro Comercial Malvinas Plaza en Lima Metropolitana? Ante la problemática se propone la solución a través de la formulación de la hipótesis: El capital de trabajo infiere en grado significativo en el desarrollo de las empresas del Centro Comercial Malvinas Plaza en Lima Metropolitana. Asimismo, este trabajo se ha orientado al siguiente objetivo: Determinar la inferencia del capital de trabajo en el desarrollo de las empresas del Centro Comercial Malvinas Plaza en Lima Metropolitana. El trabajo llega a la siguiente conclusión: Se ha determinado la inferencia del capital de trabajo en el desarrollo de las empresas del Centro Comercial Malvinas Plaza en Lima Metropolitana; mediante la gestión del efectivo y equivalentes de efectivo, cuentas por cobrar comerciales, existencias; pasivo corriente y el capital de trabajo neto de las empresas.
\end{abstract}

Palabras clave: Capital de trabajo; Desarrollo Empresarial y Centro Comercial Malvinas Plaza de Lima Metropolitana.

\section{ABSTRACT}

The problem has been identified in the deficient development of the indicated companies. This problem is expressed in the following question: How can the working capital be inferred in the development of the companies of the Malvinas Plaza Shopping Center in Metropolitan Lima? Faced with the problem, the solution is proposed through the formulation of the hypothesis: The working capital infers to a significant degree in the development of the companies of the Malvinas Plaza Shopping Center in Metropolitan Lima. Likewise, this work has been oriented to the following objective: To determine the inference of the working capital in the development of the companies of the Malvinas Plaza Shopping Center in Metropolitan Lima. The work reaches the following conclusion: The inference of the working capital in the development of the companies of the Malvinas Plaza Shopping Center in Metropolitan Lima has been determined; by managing cash and cash equivalents, trade accounts receivable, inventories; current liabilities and net working capital of companies.

Keywords: Working capital; Business Development and Malvinas Plaza de Lima Metropolitan Shopping Center.

\section{RESUMO}

O problema foi identificado no fraco desenvolvimento das empresas indicadas. Esse problema é expresso na seguinte pergunta: Como o capital de giro pode ser inferido no desenvolvimento das empresas do Shopping Center Malvinas Plaza na região metropolitana de Lima? Diante do problema, a solução é proposta através da formulação da hipótese: $O$ capital de giro infere em grau significativo no desenvolvimento das empresas do Shopping Center Malvinas Plaza na região metropolitana de Lima. Da mesma forma, este trabalho foi orientado para o seguinte objetivo: Determinar a inferência do capital de giro no desenvolvimento das empresas do Malvinas Plaza Shopping Center na região metropolitana de Lima. $\mathrm{O}$ trabalho chega à seguinte conclusão: Foi determinada a inferência do capital de giro no desenvolvimento das empresas do Malvinas Plaza Shopping Center na região metropolitana de Lima; administrando caixa e equivalentes de caixa, contas a receber de clientes, estoques; passivo circulante e capital circulante líquido das empresas.

Palavras-chave: Capital de giro; Desenvolvimento de Negócios e Centro Comercial Malvinas Plaza na Região Metropolitana de Lima. 
Introducción

El problema se ha determinado en el desarrollo de las empresas del Centro Comercial Malvinas Plaza en Lima Metropolitana de acuerdo con lo siguiente:

Síntomas: a) Deficiente gestión empresarial en términos de planeación, organización, dirección, coordinación y control de las empresas; b) Falta de economía en las empresas, al operar con altos costos y mínimos beneficios en la obtención de recursos humanos, materiales y financieros que requieren para la operativización de su giro o actividad empresarial; c) Falta de eficiencia en las empresas por la inadecuada racionalización de recursos humanos, materiales, financieros y tecnológicos y el deficiente aprovechamiento de estos en el desarrollo de su giro empresarial; d) Falta de productos y servicios de calidad, generalmente las empresas del Centro compran y venden productos chinos y peor aún se dice que son productos robados especialmente en el rubro tecnológico (celulares, laptop, computadoras, etc.).

Causas: a) En términos de gestión se ha determinado que las empresas no llevan a cabo el proceso administrativo; por tanto, no planean, organizan, dirigen, coordinan y controlan sus actividades; b) En términos de economía, no se dispone de estrategias de compras y gastos ni de obtención de mejores beneficios en las empresas; c) En términos de eficiencia no se dispone de medidas de racionalización de recursos ni de mejor aprovechamiento en las empresas; d) En términos de bienes y servicios, los mismos no se de calidad garantizada e incluso son de dudosa procedencia en las empresas.

Pronostico: a) De continuar esta situación las empresas del Centro Comercial Malvinas Plaza en Lima Metropolitana seguirán gestionándose sin planeación, organización, dirección, coordinación y control de los recursos y actividades; b) De continuar esta situación los beneficios serán cada vez menores y no se concretará el aprovechamiento de los recursos en toda su dimensión en las empresas; c) De continuar esta situación los recursos en las empresas no facilitarán la eficiencia que requieren para poder continuar en el mercado; d) De continuar esta situación no se logrará tener productos y servicios de calidad en las empresas.

Control de pronóstico: En este trabajo se proponer como alternativa de solución o herramienta para el control del nefasto pronóstico al capital de trabajo, tanto bruto como neto, el mismo que siempre debe ser positivo y alto para asegurar la operatividad de las empresas. En detalle, al respecto: a) El capital de trabajo será la herramienta financiera 
que facilitará la realización de una adecuada gestión empresarial con la planeación, organización, dirección, coordinación y control de los recursos para llevar a cabo las actividades, funciones y servicios que permitirán el desarrollo de las empresas; b) El capital de trabajo será la herramienta financiera que facilitará la economía empresarial que permitirá enrumbar al desarrollo en las empresas; c) El capital de trabajo será la herramienta financiera que facilitará la racionalización de los recursos en términos de eficiencia para llevar a cabo las actividades, funciones y procesos necesarios para el desarrollo de las empresas.

Problema general: ¿Cómo se podrá inferir el capital de trabajo en el desarrollo de las empresas del Centro Comercial Malvinas Plaza en Lima Metropolitana?

Problemas específicos: a) ¿Cómo podrá influir la gestión del efectivo y equivalente de efectivo del capital de trabajo en el desarrollo de las empresas?; b) ¿Cómo podrá incidir la gestión de las cuentas por cobrar comerciales del capital de trabajo en el desarrollo de las empresas?; c) ¿Cómo podrá influir la gestión de las existencias del capital de trabajo en el desarrollo de las empresas?; d) ¿Cómo podrá incidir la gestión del pasivo corriente del capital de trabajo neto en el desarrollo de las empresas?; e) ¿Cómo podrá influir la gestión del capital de trabajo neto en el desarrollo de las empresas?

Investigaciones nacionales: Barreda (2020) considera que la reorganización del capital de trabajo incide en alto grado en el desarrollo competitivo de las empresas del Sector Comercio de Lima Metropolitana, mediante una adecuada planeación y organización. Por su parte Rafael y Suclli (2019) consideran que el capital de trabajo es la base de toda gestión empresarial, por tanto, debe ser gestionado de la mejor manera para asegurar la operatividad empresarial, por cuanto dicha operatividad no será posible sin tener un buen capital de trabajo. Entre tanto, Uriarte (2018) considera que las empresas necesitan tener liquidez para cumplir con las obligaciones de corto plazo, tales como deudas con trabajadores, proveedores, estado, acreedores, ect. Por su parte Alderete (2016) concluye que las decisiones financieras, especialmente las referidas a capital de trabajo facilitarían el desarrollo de las pequeñas y medianas empresas; mediante las decisiones de financiamiento, inversión, rentabilidad y riesgos tomadas en forma oportuna y con la información suficiente, competente y relevante. Sin embargo, Osorio (2018) considera que la administración financiera se relaciona con el desarrollo de las medianas empresas de servicios de Lima Metropolitana; mediante las decisiones de financiamiento, decisiones de inversiones (como las de capital de trabajo), decisiones de 
rentabilidad y decisiones sobre riesgos de las empresas indicadas. De otro lado, Murillo (2018) concluye que la gestión del talento humano facilita el desarrollo de las empresas; mediante la planeación, organización y evaluación de dicho talento de tal modo que aporte lo mejor de sí para concretar el desarrollo de las empresas las empresas; siendo que dicho desarrollo significa eficiencia, efectividad, mejora continua, productividad y competitividad.

Investigaciones internacionales: Berrezueta (2018) considera que la administración financiera desempeña un rol muy relevante en la operatividad de empresas exitosas, asignando fuentes de financiamiento para el capital de trabajo de las empresas. Feldman (2018) considera que la gestión del capital de trabajo es esencial en toda actividad económica, cualquiera sea su tamaño o sector empresarial; por cuanto de nada vale tener bienes de capital si no se tiene el capital de trabajo para que todo opere, se movilice, marche la empresa. Vásquez (2016) considera que el capital de trabajo neto es una herramienta útil a la hora de evaluar la liquidez de la empresa; siendo que la mayor cantidad de capital de trabajo bruto frente a una mejor cantidad de pasivo corriente, pone a la empresa en capacidad de pago de sus obligaciones de corto plazo. De otro lado, Peñafiel (2016) el capital de trabajo tiene que planearse, organizarse, dirigirse, coordinarse, controlarse y retroalimentarse continuamente de tal modo que se cuenten con los activos corrientes necesarios y que sean mayores al pasivo corriente de este tipo de empresas. Namicela. y Quituisaca (2016) indican que el capital de trabajo neto es la diferencia del activo corriente menos el pasivo corriente. $Y$ en cuanto al índice de capital de trabajo se tiene al activo corriente entre el pasivo corriente, comúnmente llamado índice de liquidez general; al respecto tanto el capital de trabajo neto como elo índice de capital de trabajo deben ser razonables de tal modo que aseguren la operatividad empresarial. Márquez (2017) concluye que la sostenibilidad en las empresas está relacionada con el desarrollo empresarial que logren y este a su vez está ligado a la responsabilidad social corporativa (RSC); sin desarrollo empresarial no se puede tener eficiencia ni efectividad; menos mejora continua ni productividad; por lo que es imprescindible sentar las base para un fuerte y sostenido desarrollo empresarial. Rodríguez (2015) considera que la relación entre el capital de trabajo y los procesos de desarrollo ha sido uno de los temas centrales de análisis de las investigaciones del capital de trabajo; por cuanto no se puede lograr un adecuado desarrollo si las empresas no cuentan el capital de trabajo que les permita funcionar; el capital de trabajo tiene los 
recursos para la operatividad y esta es la base para comprar, producir, vender y mover el aparato empresarial sentando las bases para una buena gestión de las deudas como las inversiones empresariales como base para el desarrollo empresarial.

\section{Bases teóricas:}

Capital de trabajo: Según Weston (2018) los activos o inversiones de una empresa se dividen en capital de trabajo bruto y bienes de capital. Siendo el capital de trabajo bruto igual al activo corriente; y, los bienes de capital igual al activo no corriente. En buena cuenta el capital de trabajo está conformado por los bienes y derechos de corto plazo, siendo este menor a un año calendario. De otro lado, Van Horne (2018) el capital de trabajo bruto está conformado por el activo corriente de las empresas; mientras que el capital de trabajo neto está conformado por la diferencia entre el activo corriente menos el pasivo corriente. Por su parte Gitman (2016) considera que el capital de trabajo o fondo de maniobra (también denominado capital circulante, capital corriente, fondo de rotación o capital de rotación), es la parte del activo circulante que es financiada con recursos de carácter permanente; siendo que más específicamente corresponde a la diferencia del activo corriente menos el pasivo corriente. Entre tanto Besley y Brigham (2018) considera que el capital de trabajo o fondo de maniobra es el rubro de la gestión financiera empresarial que tiene en cuenta al activo corriente que es necesario para llevar a cabo las actividades propias del giro que desarrolla cada empresa. Según Gómez (2019) el capital de trabajo neto de las empresas se establecer como la diferencia que se presenta entre los activos corrientes o de corto plazo menos los pasivos corrientes o de corto plazo de dichas empresas. Por su parte, Gitman y Zutter (2016) consideran que el capital de trabajo constituye el conjunto de recursos, bienes y derechos, de corto plazo, menor a un año y fácilmente convertibles en efectivo y equivalentes de efectivo, que necesitan las empresas para llevar a cabo su giro o actividad en un determinado periodo de funcionamiento empresarial.

Desarrollo empresarial: Según Koontz \& O’Donnell (2017): El desarrollo empresarial está referido al progreso que experimenta la empresa como consecuencia de su evolución positiva a lo largo del tiempo; es decir es el corolario de una situación gestionada con anterioridad. Según Díaz (2018) el desarrollo empresarial, es el nivel de progreso, optimización o perfeccionamiento de la gestión empresarial; $\mathrm{y}$ de haber trabajado para tener determinados atributos como el de la economía, eficiencia, efectividad, mejora continua y competitividad que deben tener las empresas en un 
determinado período; para lo cual las empresas deben disponer de estándares o indicadores para poder comparar lo que vienen haciendo. Para Flores (2018) el desarrollo empresarial se logra con una buena situación financiera; es decir, estructurando adecuadamente las fuentes de financiamiento que permitan asumir menores costos; y, haciendo que las inversiones, tanto corrientes como no corrientes le den a la empresa un alto grado de rentabilidad. Según Johnson \&Scholes (2018) considera que el desarrollo empresarial es una condición que pueden lograr las empresas después de tener una adecuada gestión empresarial, la misma que considera a la planeación, organización, dirección, coordinación, integración, control e incluso la retroalimentación que resulta necesaria para concretar las actividades empresariales con sus funciones y procesos, que permiten atender la demanda que se tiene en determinado nicho del mercado. En el mismo sentido, Pérez (2018) considera que el desarrollo empresarial es una posición que pueden lograr las empresas con mucho esfuerzo y dedicación. En la óptica de Murcia (2018) el desarrollo empresarial se logra con buenos planes, organización de sus recursos, amplios conocimientos para tomar decisiones acertadas, coordinando con los grupos de interés y controlando todo el quehacer empresarial. Para Robbins \& Coulter (2015) el desarrollo empresarial es la consecuencia de un proceso empresarial llevado a cabo con los recursos más adecuados posibles, con la gestión de mayor eficiencia posible y desde luego con una buena administración de todo el acontecer empresarial.

Centro Comercial Malvinas Plaza de Lima Metropolitana: Según Aranda (2017): Un centro comercial1 (en inglés: shopping mall o shopping center) es una construcción que consta de uno o varios edificios, por lo general de gran tamaño, que albergan servicios, locales y oficinas comerciales aglutinados en un espacio determinado concentrando mayor cantidad de clientes potenciales dentro del recinto. Según Radio Programas del Perú (30 de enero del 2020) el Módulo de Seguridad Ciudadana está ubicado en la Av. Argentina y el cruce con el Jr. Cárcamo, punto estratégico desde el que se vigilará, durante las 24 horas del día.

\section{Marco conceptual:}

Gestión del efectivo y equivalentes de efectivo: De acuerdo con la Dirección Nacional de Contabilidad Pública (2019) el efectivo y equivalentes de efectivo agrupa al dinero en efectivo, cheques, giros, entre otros, así como los depósitos en instituciones financieras, y otros equivalentes de efectivo disponibles a requerimiento del titular. 
Gestión de las cuentas por cobrar comerciales: De acuerdo con la Dirección Nacional de Contabilidad Pública (2019); las Cuentas por cobrar comerciales - terceros; representan los derechos de cobro a terceros que se derivan de las ventas de bienes y/o servicios que realizan las empresas debido a su objeto de negocio.

Gestión de las existencias: De acuerdo con la Dirección Nacional de Contabilidad Pública (2019); las existencias de las empresas también se les llama bienes de cambio porque son adquiridas para cambiarlas, es decir venderlas o consumirlas en el corto plazo.

Gestión del activo corriente: Según Heredia (2014) el activo corriente forma parte del activo total de una empresa y está compuesto por los activos más líquidos de la empresa.

Gestión del pasivo corriente: Según Heredia (2014): se incluyen dentro de esta clasificación todas aquellas partidas que representen obligaciones de la empresa cuyo pago debe efectuarse durante su ciclo operacional (corto plazo).

Gestión del capital de trabajo bruto: Según Heredia (2018) el capital de trabajo bruto es igual al importe del Activo Corriente del Estado de situación financiera de las empresas, este punto de vista nos hace apreciar al capital de trabajo con carácter cualitativo.

Gestión del capital de trabajo neto: Según Heredia (2018) el Capital de Trabajo neto es igual a activo corriente (plazo de conversión máximo de un año) menos el pasivo corriente a corto plazo (plazo de pago máximo de un año) con que cuenta la empresa.

Proceso de gestión empresarial: Es el conjunto de fases para llevar a cabo la gestión de las empresas. A continuación, se describen las fases respectivas:

Planeación: Para Robbins \& Coulter (2018) la planeación es la determinación anticipada de los recursos, actividades y procesos que van a desarrollar las empresas para llevar a cabo su giro en un determinado período.

Organización.: Para Robbins \& Coulter (2018) se refiere a distribuir o señalar las necesarias actividades de trabajo entre los miembros del grupo e indicar la participación de cada miembro del grupo.

Ejecución: Para Robbins \& Coulter (2018) para llevar a cabo físicamente las actividades que resulten de los pasos de planeación y organización, es necesario que el 
gerente tome medidas que inicien y continúen las acciones requeridas para que los miembros del grupo ejecuten la tarea.

Control: Para Robbins \& Coulter (2018) los gerentes siempre han encontrado conveniente comprobar o vigilar lo que sé está haciendo para asegurar que el trabajo de otros está progresando en forma satisfactoria hacia el objetivo predeterminado.

Economía y eficiencia empresarial: La economía y la eficiencia son dos importantes atributos del desarrollo empresarial y cuyo concepto se detalla a continuación:

Economía empresarial: Para Chiavenato (2016) la economía empresarial está relacionada con las adquisiciones de bienes y servicios a los menores costos posibles y con el propósito de obtener de los mismos el mayor beneficio. En este contexto entra en juego la expresión económica: Costo-Beneficio.

Eficiencia empresarial: Para Chiavenato (2014) la eficiencia empresarial, es sinónimo de hacer bien todo lo que corresponde a una empresa; también es el resultado positivo luego de la racionalización adecuada de los recursos, acorde con la finalidad buscada por los responsables de la gestión de las empresas.

Productos y servicios de calidad: De acuerdo con Steiner (2017) las empresas comerciales como las empresas de servicios deben comprar mercaderías de una calidad garantizada para asegurar su venta y generar confianza en los clientes.

Productividad empresarial: De acuerdo con Steiner (2017) la productividad empresarial es el retorno que producen las acciones llevadas a cabo por una empresa.

Mejora continua empresarial: Para Robbins \& Coulter (2018) la mejora continua se orienta a lograr economía, eficiencia y efectividad. Para Johnson \& Scholes (2018) la mejora continua empresarial, se concreta con la reducción de costos y gastos, racionalización, mejor aprovechamiento de los recursos; logro de metas, objetivos. Según la opinión de Thompson (2018) la mejora continua es la relación entre producción final y los factores utilizados en la producción de bienes y servicios. La mejora continua es sinónimo de productividad.

Justificación metodológica: Metodológicamente este trabajo se justifica porque en primer lugar se ha identificado el problema y sobre el mismo se ha establecido su 
planteamiento, descripción, formulación e incluso sus relaciones con el marco teórico y otros elementos de la investigación.

Objetivo general: Determinar la inferencia del capital de trabajo en el desarrollo de las empresas del Centro Comercial Malvinas Plaza en Lima Metropolitana.

Objetivos específicos: a) Establecer la influencia de la gestión del efectivo y equivalente de efectivo del capital de trabajo en el desarrollo de las empresas; b) Determinar la incidencia de la gestión de las cuentas por cobrar comerciales del capital de trabajo en el desarrollo de las empresas; c) Establecer la influencia de la gestión de las existencias del capital de trabajo en el desarrollo de las empresas d) Determinar la incidencia de la gestión del pasivo corriente del capital de trabajo neto en el desarrollo de las empresas; e) Establecer la influencia de la gestión del capital de trabajo neto en el desarrollo de las empresas.

Hipótesis general: El capital de trabajo infiere en grado significativo en el desarrollo de las empresas del Centro Comercial Malvinas Plaza en Lima Metropolitana.

Hipótesis específicas; a) La gestión del efectivo y equivalente de efectivo del capital de trabajo influye en grado significativo en el desarrollo de las empresas; b) La gestión de las cuentas por cobrar comerciales del capital de trabajo incide en grado significativo en el desarrollo de las empresas; c) La gestión de las existencias del capital de trabajo influye en grado significativo en el desarrollo de las empresas; d) La gestión del pasivo corriente del capital de trabajo neto incide en grado significativo en el desarrollo de las empresas; e) La gestión del capital de trabajo neto influye en grado significativo en el desarrollo de las empresas.

\section{Materiales y métodos}

Tipo de investigación: Es de tipo aplicada. Por cuanto se propone aplicar el capital de trabajo de las empresas para solucionar el problema del deficiente desarrollo que enfrentan más aún en un contexto pandémico y de cambios de gobierno que se vive en el país.

Diseño de la investigación: El diseño que se aplicó fue el no experimental; dicho diseño se configura considerando que no es necesario aplicar las teorificaciones vertidas por un lado y por otro no manipulando ni controlando losa resultados obtenido de los participantes en el trabajo realizado 
Población: Fueron 57 las personas consideradas en la población de investigación y que están relacionadas y mostraron su disposición para colaborar sobre el capital de trabajo y el desarrollo empresarial

Muestra de la investigación: Fueron 50 las personas consideradas en la muestra del trabajo, obtenidas aleatoriamente y aplicando la fórmula para poblaciones menores a cien mil, según la teoría estadística generalmente aceptada.

Área de estudio: Este trabajo se ha realizado en las empresas del Centro Comercial Malvinas Plaza en Lima Metropolitana, un famoso lugar comercial de emprendedores populares que a punta de esfuerzo sacan adelante sus negocios.

Procedimientos estadísticos: El capital de trabajo y el desarrollo de las empresas del Centro Comercial Malvinas Plaza en Lima Metropolitana; ha aplicado varios procedimientos estadísticos para contrastar la hipótesis, mediante la Correlación de Spearman de las variables del trabajo, como para determinar el Grado de confianza y rechazar la hipótesis nula de cada una de las contrastaciones realizadas.

Aspectos éticos: Para Muñoz (2018) la mayor responsabilidad de la empresa es crear riqueza y contribuir a la prosperidad económica de la sociedad en la que vive y para ello la mejor manera de garantizar su supervivencia es actuar ética y responsablemente. Por lo demás en ste trabajo todo lo expresado es la verdad.

\section{Resultados}

a) El $90 \%$ de los encuestados acepta que financieramente el capital de trabajo es el conjunto de recursos que necesitan para operar rutinariamente las empresas.

b) El 90\% de los encuestados acepta que el efectivo y equivalente de efectivo es un principal componente del capital de trabajo de las empresas.

c) El 84\% de los encuestados acepta que el efectivo y equivalentes de efectivo está compuesto por dinero en caja, fondo fijo, cuenta corriente, cuenta a plazos y otras modalidades que utilicen las empresas.

d) El $85 \%$ de los encuestados acepta que las cuentas por cobrar son derechos componentes del capital de trabajo fácilmente convertibles en efectivo en las empresas. 
e) El $90 \%$ de los encuestados acepta que las cuentas por cobrar comerciales deben gestionarse eficientemente para evitar provisionarlas y perder la capacidad de recuperarlas en las empresas.

f) El 90\% de los encuestados acepta que el rubro existencias comprende las mercaderías y es uno de los más importantes para las empresas.

g) El $90 \%$ de los encuestados acepta que las mercaderías deben ser gestionadas eficientemente en sus entradas, salidos y saldos para evitar estar por encima o debajo del stock mínimo en las empresas.

h) El 67\% de los encuestados acepta que el pasivo corriente comprende las deudas de corto plazo y sirve para determinar el capital de trabajo neto de las empresas

i) El 96\% de los encuestados acepta que el pasivo corriente está conformado por deudas laborales, tributarias, comerciales, financieras y otras; y que deben ser las menores posibles en las empresas.

j) El 91\% de los encuestados acepta que el capital de trabajo neto es la resta del activo corriente menos el pasivo corriente en las empresas.

k) El 83\% de los encuestados acepta que se debe procurar el capital de trabajo neto sea positivo para augurar una buena operatividad en las empresas.

1) El $89 \%$ de los encuestados acepta que el desarrollo empresarial es un proceso que comprende la gestión, economía y eficiencia, como los productos y servicios de calidad, logrando productividad y mejora continua de las empresas.

m) El 85\% de los encuestados acepta que el proceso de gestión empresarial comprende la planeación, organización, dirección, coordinación y control de los recursos y operaciones de las empresas.

n) El $88 \%$ de los encuestados acepta que el proceso de gestión empresarial debe llevarse a cabo en base a políticas, estrategias y acciones para operar en las mejores condiciones las empresas.

o) El $89 \%$ de los encuestados acepta que la economía empresarial es un aspecto básico y consiste en gestionar con bajos costos y mayores beneficios a las empresas.

p) El $88 \%$ de los encuestados acepta que la eficiencia empresarial se refiere a la racionalización de los recursos y al mejor aprovechamiento de estos en las operaciones de las empresas. 
q) El $84 \%$ de los encuestados acepta que los productos deben ser de calidad certificada para asegurar el desarrollo de las empresas.

r) El 85\% de los encuestados acepta que además de los productos se deben agregar determinados servicios igualmente de calidad para desarrollar efectivamente las empresas.

s) El 84\% de los encuestados acepta que la productividad empresarial en general es el mejor aprovechamiento de los recursos disponibles en las empresas.

t) El $85 \%$ de los encuestados acepta que la productividad empresarial se refiere a la cantidad de productos negociados y los recursos empleados en las empresas.

u) El 84\% de los encuestados acepta que la mejora continua en esencia se refiere a mejorar los recursos, actividades, procesos y procedimientos de las empresas.

v) Al analizar la respuesta se llegó a determinar que el $85 \%$ de los encuestados acepta que la mejora continua se refiere a la reducción de costos, gastos y pérdidas para obtener mayor rentabilidad en las empresas.

Contrastación de la hipótesis general: La correlación es igual a 90.45\%, lo cual indica correlación directa, alta y por tanto apoya el modelo de investigación desarrollada. Por su parte el valor de significancia fue igual a 3.39\%, con lo cual se rechaza la hipótesis nula y se acepta la hipótesis alternativa. Además, se tuvo una regresión línea del 81.81\%, lo cual indica el peso que tiene el capital de trabajo en el desarrollo empresarial.

Contrastación de la hipótesis específica a): El valor de la correlación es igual a $90.38 \%$, lo cual indica correlación directa, alta y por tanto apoya el modelo de investigación desarrollada. El valor de significancia, igual a 3.32\% permitió rechazar la hipótesis nula y aceptar la alternativa. Asimismo, se determinó la regresión lineal igual a $81.69 \%$, dicho valor indica el peso que tiene la gestión del efectivo y equivalente de efectivo del capital de trabajo en el desarrollo empresarial, lo cual es cierto, por cuanto sin los recursos financieros es poco lo que se puede hacer.

Contrastación de la hipótesis específica b): La correlación es igual a 90.12\%, lo cual indica correlación directa, alta y por tanto apoya el modelo de investigación desarrollada. El valor de significancia igual a 4.12\% y la correlación lineal igual a $81.22 \%$, lo cual indica el peso que tiene la gestión de las cuentas por cobrar comerciales del capital de trabajo en el desarrollo empresarial. 
Contrastación de la hipótesis específica c): La correlación es igual a 90.33\%, lo cual indica correlación directa, alta y por tanto apoya el modelo de investigación desarrollada. El valor de significancia igual a 4.05\% permitió rechazar la hipótesis nula y la regresión lineal del $81.60 \%$, lo cual explica el peso que tiene la gestión de las existencias del capital de trabajo en el desarrollo de las empresas.

Contrastación de la hipótesis específica d): La correlación es igual a 90.05\%, lo cual indica correlación directa, alta y por tanto apoya el modelo de investigación desarrollada. El valor de significancia igual a 3.16\% que permitió rechazar la hipótesis nula; y la regresión lineal del $81.09 \%$ que establece la influencia de la gestión del pasivo corriente del capital de trabajo neto en el desarrollo de las empresas.

Contrastación de la hipótesis específica e): El valor de la correlación es igual a $90.15 \%$, lo cual indica correlación directa, alta y por tanto apoya el modelo de investigación desarrollada. El valor de significancia igual a $3.55 \%$ permitió aceptar la hipótesis alternativa; además se tiene que la regresión lineal igual a 81.27\% muestra un alto peso o influencia de la gestión del capital de trabajo neto en el desarrollo de las empresas.

\section{Discusión}

a) En el marco para determinar la inferencia del capital de trabajo en el desarrollo de las empresas del Centro Comercial Malvinas Plaza en Lima Metropolitana se tiene que el $90 \%$ de los encuestados acepta que financieramente el capital de trabajo es el conjunto de recursos que necesitan para operar rutinariamente las empresas del Centro Comercial Malvinas Plaza en Lima Metropolitana. Este resultado es similar al 91\% presentado, aunque en otra dimensión espacial y temporal, por Barreda, N. (2019). El capital de trabajo y el desarrollo competitivo de las Empresas del Sector Comercio de Lima Metropolitana. [Tesis de maestría, Universidad Nacional Federico Villarreal]. Dicho trabajo se encuentra en el Repositorio institucional. Al respecto se tiene que ambos trabajos tienen resultados altos, lo cual favorece el cumplimiento del objetivo y la realización del trabajo en general.

b) En el contexto para determinar la inferencia del capital de trabajo en el desarrollo de las empresas del Centro Comercial Malvinas Plaza en Lima Metropolitana, se tiene que el $89 \%$ de los encuestados acepta que el desarrollo empresarial es un proceso que 
comprende la gestión, economía y eficiencia, como los productos y servicios de calidad, logrando productividad y mejora continua de las empresas del Centro Comercial Malvinas Plaza en Lima Metropolitana. Este resultado es similar al 90\% presentado, aunque en otra dimensión espacial y temporal, por Alderete, J. (2016). Las decisiones financieras y el desarrollo de las pequeñas y medianas empresas de confecciones textiles del Emporio Comercial Gamarra de Lima Metropolitana. (Tesis Doctoral). Universidad Nacional Federico Villarreal. Dicho trabajo se encuentra en la biblioteca institucional. Ambos resultados son altos y favorecen el modelo de investigación desarrollado.

\section{Conclusiones}

a) Se ha determinado la inferencia del capital de trabajo en el desarrollo de las empresas; mediante la gestión del efectivo y equivalentes de efectivo, cuentas por cobrar comerciales, existencias; pasivo corriente y el capital de trabajo neto de las empresas.

b) Se ha establecido la influencia de la gestión del efectivo y equivalente de efectivo del capital de trabajo en el desarrollo de las empresas; mediante la gestión eficiente de caja, cuentas corrientes, cuentas a plazos, cuentas de ahorros, certificados bancarios y otros instrumentos financieros que utilizan las empresas.

c) Se ha determinado la incidencia de la gestión de las cuentas por cobrar comerciales del capital de trabajo en el desarrollo de las empresas, mediante la identificación de las cuentas en cobranza dudosa, la provisión de las cuentas incobrables, el castigo o eliminación en los libros contables y si fuera el caso la recuperación extraordinaria de dichas cuentas.

d) Se ha establecido la influencia de la gestión de las existencias del capital de trabajo en el desarrollo de las empresas mediante la gestión de las entradas, salidas y saldos de las mercaderías de estas empresas.

e) Se ha determinado la incidencia de la gestión del pasivo corriente del capital de trabajo neto en el desarrollo de las empresas; mediante la gestión de las deudas de corto plazo de tipo laboral, tributario, comercial, financiero y otras cuentas por pagar de las empresas.

f) Se ha establecido la influencia de la gestión del capital de trabajo neto en el desarrollo de las empresas; mediante la gestión eficiente del activo corriente como el pasivo corriente de este tipo de empresas. 


\section{Recomendaciones}

a) Se recomienda a los propietarios, socios o accionistas; como administradores tener en cuenta que el capital de trabajo infiere en grado significativo en el desarrollo de las empresas. Por tanto, debe disponerse en cada período del capital de trabajo que permita operar las empresas sin ninguna dificultad, porque es el motor que mueve los negocios.

b) Se recomienda a los propietarios, socios o accionistas; como administradores tener en cuenta que la gestión del efectivo y equivalente de efectivo del capital de trabajo influye en grado significativo en el desarrollo de las empresas. Por tanto, se debe planear y organizar en forma eficiente las distintas cuentas que corresponden al efectivo y equivalentes de efectivo de las empresas.

c) Se recomienda a los propietarios, socios o accionistas; como administradores tener en cuenta que la gestión de las cuentas por cobrar comerciales del capital de trabajo incide en grado significativo en el desarrollo de las empresas. Por tanto, debe planearse, organizarse y controlarse todos los aspectos que involucra a dichas cuentas por cobrar comerciales para evitar que se vuelvan irrecuperables.

d) Se recomienda a los propietarios, socios o accionistas; como administradores tener en cuenta que la gestión de las existencias del capital de trabajo influye en grado significativo en el desarrollo de las empresas. Por tanto, debe coordinarse con los proveedores para disponer del stock indispensable para atender a los clientes.

e) Se recomienda a los propietarios, socios o accionistas; como administradores tener en cuenta que la gestión del pasivo corriente del capital de trabajo neto incide en grado significativo en el desarrollo de las empresas. Por tanto, se debe negociar las deudas de corto plazo con los diversos agentes económicos, tales como trabajadores, Estado, proveedores y acreedores.

f) Se recomienda a los propietarios, socios o accionistas; como administradores tener en cuenta que la gestión del capital de trabajo neto influye en grado significativo en el desarrollo de las empresas. Por tanto, debe planearse la disponibilidad adecuada de capital de trabajo neto, considerando que este se constituye de restar activo corriente menos pasivo corriente.

\section{Referencias}

Alderete, J. (2016). Las decisiones financieras y el desarrollo de las pequeñas y medianas empresas de confecciones textiles del Emporio Comercial Gamarra de Lima 
Metropolitana. [Tesis doctoral, Universidad Nacional Federico Villarreal]. Biblioteca EUPG-UNFV.

Aranda, A. (2017). Los centros comerciales de Estados Unidos se vacían - Un símbolo de la vida americana se tambalea. El gran espacio comercial que conocemos se hace viejo y necesita reinventarse - Internet y un nuevo consumidor son las palancas de su renovación. Publicado por Forbes, 20 de julio de 2017. Recuperado de: https://es.wikipedia.org/wiki/Centro_comercial

Barreda, N. (2019). El capital de trabajo y el desarrollo competitivo de las Empresas del Sector Comercio de Lima Metropolitana. [Tesis de maestría, Universidad Nacional Federico Villarreal]. Repositorio institucional: http://repositorio.unfv.edu.pe/handle/unfv/4179

Berrezueta, M. (2018). La gestión del capital de trabajo y su impacto en la rentabilidad en las empresas de la ciudad de cuenca del sector productivo de elaboración de productos alimenticios. [Tesis de pregrado, Universidad Politécnica Salesiana]. Repositorio institucional: https://dspace.ups.edu.ec/bitstream/123456789/16101/1/ups-ct007795.pdf

Besley, S. y Brigham, E. (2018). Fundamentos de Administración Financiera. Editorial Harper \& Row Latinoamericana.

Chiavenato, I. (2016) Teoría general de la administración. Mc. Graw Hill Interamericana SA.

Díaz, R. (2018). Calidad total en la empresa peruana. Fondo de Desarrollo Editorial de la Universidad de Lima.

Dirección Nacional de Contabilidad Pública (2019). Plan Contable General Empresarial. El Peruano. Recuperado de: https://www.mef.gob.pe/contenidos/conta_publ/documentac/version_modificada_ pcg_empresarial.pdf

Feldman, G. (2018). El capital de trabajo en las pequeñas y medianas empresas como restricción a su operatoria. [Artículo Científico, Universidad de Buenos Aires]. Repositorio institucional: ppct.caicyt.gov.ar/rain/article/download/pdf

Flores, J. (2018). Gestión Financiera: Teoría y Práctica. Editorial CECOF Asesores. 
Gitman J. (2016). Administración Financiera moderna. Editorial Harper \& Row Latinoamericana.

Gómez, G. (2019). Administración del capital de trabajo. Editorial Continental

Heredia, D. (2014). Administración del capital de trabajo de las empresas. Editorial San Juan.

Johnson, G. \& Scholes, K. (2018). Dirección Estratégica. Prentice May International.

Koontz, H. \& O’Donnell, C. (2017). Administración Moderna. Litográfica Ingramex

Márquez, M. (2017). Sostenibilidad, comunicación y valor compartido: el discurso actual del desarrollo sostenible en la empresa española. [Tesis doctoral, Universidad Complutense de Madrid]. Repositorio institucional: https://eprints.ucm.es/42084/1/T38628.pdf

Muñoz, J. (2018). Ética empresarial, Responsabilidad Social Corporativa (RSC) y Creación de Valor Compartido (CVC). [Artículo científico, Universidad Complutense de Madrid]. Repositorio institucional: http://ojsuniversia.xercode.com/index.php/gcg/article/view/457/etica-empresarialresponsabilidad-social-corporativa-rsc-creacion-valor-compartido-cvc-

Murcia, H. (2018). Creatividad e innovación para el desarrollo empresarial. Días De Santos.

Murillo, D. (2018). La gestión del talento humano y el desarrollo de las empresas industriales de confecciones de Lima Metropolitana, análisis y propuesta actual. [Tesis de maestría, Universidad Nacional Federico Villarreal]. Repositorio institucional: http://repositorio.unfv.edu.pe/handle/unfv/2474

Namicela, D. y Quituisaca, D. (2016). Diseño y elaboración de una guía para la gestión del capital de trabajo para las cooperativas de ahorro y crédito no sujetas al control de la superintendencia de bancos y seguros de la ciudad de Loja. [Tesis de maestría, Universidad Nacional de Loja]. Repositorio institucional http://dspace.unl.edu.ec/jspui/bitstream/123456789/1672/1/tesis.pdf

Osorio, M. (2018). La administración financiera y el desarrollo de las medianas empresas de servicios de Lima Metropolitana, Período 2014-2016. [Tesis de maestría, Universidad Nacional Federico Villarreal]. Repositorio institucional: http://repositorio.unfv.edu.pe/handle/unfv/2074 
Peñafiel, J. (2016). Administración del capital de trabajo y su incidencia en la rentabilidad de la fábrica de aluminios hércules. [Tesis de pregrado, Universidad Técnica de Ambato]. Repositorio institucional: http://repositorio.uta.edu.ec/bitstream/123456789/20813/1/T2744i.pdf

Pérez, E. (2018). Organización y Administración de empresas. Editorial San Marcos.

Radio Programas del Perú (30 de enero del 2020). Centro Comercial "Malvinas Plaza" garantiza seguridad para todos. Lima. Recuperado de: https://rpp.pe/lima/actualidad/centro-comercial-malvinas-plaza-garantizaseguridad-para-todos-noticia-309240

Rafael, V. y Suclli, H. (2019). Capital de Trabajo y su incidencia en el crecimiento económico de la empresa Cap Magic S.A.C. en la industria textil del sector de confección de gorros en el emporio de Gamarra en el periodo 2013-2017. [Tesis de pregrado, Universidad Tecnológica del Perú]. Repositorio institucional: http://repositorio.utp.edu.pe/bitstream/utp/2197/1/victor\%20rafael_henry\%20sucll i_trabajo\%20de\%20investigacion_bachiller_2019.pdf

Ramos, M. (2019). Análisis de capital de trabajo en una empresa de servicios, Lima 2019. [Tesis de pregrado, Universidad Norbert Wiener]. Repositorio institucional: http://repositorio.uwiener.edu.pe/bitstream/handle/123456789/3607/t061_471417 76_b.pdf? sequence $=1 \&$ isallowed $=\mathrm{y}$

Robbins, S. y Coulter, M. (2015). Fundamentos de Administración. Prentice Hall Hispanoamericana.

Robbins, S. y Coulter, M. (2018). Administración. Prentice Hall Hispanoamericana.

Rodríguez, P. (2015) El capital social como factor de innovación y desarrollo empresarial en Andalucía. [Tesis doctoral, Universidad de Sevilla]. Repositorio institucional: https://www.researchgate.net/publication/261841667_El_capital_social_como_fac tor_de_innovacion_y_desarrollo_empresarial_en_Andalucia

Steiner, G. (2017). Planeación Estratégica. Compañía Editorial Continental.

Thompson, I. (2018). Mejoramiento empresarial. México: Compañía Editorial Continental.

Uriarte, L. (2018). Gestión financiera para mejorar la liquidez de una empresa de servicios de transporte, Lima 2018. [Tesis de pregrado, Universidad Norbert 
Wiener].

Recuperado

de:

http://repositorio.uwiener.edu.pe/bitstream/handle/123456789/2686/tesis\%20uriar te $\% 201$ uzbeni.pdf?sequence $=1 \&$ isallowed $=y$

Van Horne, W. (2018). Administración financiera. Mc Graw Hill

Vásquez, M. (2016). Propuesta de manejo de gestión financiera del capital de trabajo en una compañía de seguros, Sweaden SA. [Tesis de pregrado, Pontificia Universidad Católica]. Recuperado

de: http://repositorio.puce.edu.ec/bitstream/handle/22000/8116/tesis $\% 20$ propuesta $\% 2$ 0de $\% 20$ manejo $\% 20 \mathrm{de} \% 20$ gesti $\%$ c3\%93n $\% 20$ financiera $\% 20 \mathrm{del} \% 20 \mathrm{capital} \% 20 \mathrm{de}$ \%20trabajo\%20en\%20una\%20compa\%c3\%91\%c3\%8da\%20de\%20seguros.pdf?s equence $=1$

Weston, J. (2018). Administración financiera. Mc Graw Hill 